\title{
Safety, Efficacy and Pharmacokinetics of a New 10\% Liquid Intravenous Immunoglobulin (IVIG) in Patients with Primary Immunodeficiency
}

\author{
Richard L. Wasserman • Joseph A. Church • \\ Mark Stein • James Moy • Martha White • \\ Steven Strausbaugh • Harry Schroeder • Mark Ballow • \\ James Harris • Isaac Melamed • David Elkayam • \\ William Lumry • Daniel Suez • Syed M. Rehman
}

Received: 4 November 2011 / Accepted: 18 January 2012 /Published online: 6 March 2012

(C) The Author(s) 2012. This article is published with open access at Springerlink.com

adverse events during infusion, and up to $72 \mathrm{~h}$ after infusion, including those unrelated to study product, was $27.7 \%$ with an upper $95 \%$ confidence limit $\leq 30.6 \%$. Two serious bacterial infections (SBIs) were observed resulting in a serious bacterial infection rate of 0.035 per person per year and an upper one-sided $99 \%$ confidence limit of $\leq 0.136 \mathrm{SBI} / \mathrm{pa}-$ tient/year. The number of days of work or school missed due to infection were relatively low at 2.28 days/patient/year.

M. Ballow

Women \& Children's Hospital of Buffalo,

Buffalo, NY, USA

J. Harris

South Bend Clinic LLP,

South Bend, IN, USA

I. Melamed

1st Allergy \& Clinical Research Center,

Centennial, CO, USA

D. Elkayam

Bellingham Asthma, Allergy \& Immunology Clinic,

Bellingham, WA, USA

W. Lumry

Allergy and Asthma Specialists,

Dallas, TX, USA

D. Suez

Allergy, Asthma \& Immunology Clinic, PA,

Dallas, TX, USA

S. M. Rehman

Allergy \& Asthma Center,

Toledo, OH, USA 
Two patients were hospitalized for infection producing a rate of 0.21 hospitalization days/patient/year. The IgG half-life was approximately 30 days with variation among individuals.

Conclusions Pharmacokinetic parameters of specific antibody activities were essentially the same as those of total IgG. Biotest-IVIG is safe and effective in the treatment of PID.

Keywords Intravenous immunoglobulin (IVIG) · primary immunodeficiency (PID) · clinical trial · safety · efficacy · pharmacokinetics

\section{Introduction}

Human immunoglobulin $\mathrm{G}$ (IgG) has been used to treat people with inherited antibody deficiencies since 1952 when Bruton demonstrated that monthly subcutaneous injections of IgG successfully prevented recurrent pneumococcal infections in a child with agammaglobulinemia [1]. The first commercial human IgG preparations were restricted to intramuscular injection at relatively low doses, usually $25 \mathrm{mg} / \mathrm{kg} /$ week. In order to avoid the pain of intramuscular IgG injections and to administer larger doses, intravenous immunoglobulin products (IVIG) were developed. Periodic reports of hepatitis transmission and recognition that primary immunodeficient (PID) patients must be treated for a lifetime resulted in development of IVIG manufacturing procedures that inactivate or remove blood-borne pathogens. Manufacturing steps that enhance purity, minimize damage to $\mathrm{IgG}$ molecules, decrease the frequency of adverse reactions, and result in higher concentration liquid IVIG have also been introduced. We report here the results of a clinical trial of a new liquid 10\% IVIG (Biotest-IVIG) in patients with primary immunodeficiency.

\section{Methods}

\section{Study Product}

The investigational 10\% liquid IVIG used in this study (Biotest-IVIG) was manufactured by Nabi Biopharmaceuticals, subsequently acquired by Biotest Pharmaceuticals Corporation, in Boca Raton, Florida. Biotest-IVIG was manufactured from source plasma, fully screened for blood borne pathogens, by Cohn-Oncley fractionation followed by ion exchange chromatography $[2,3]$. Three virus removal/ inactivation steps (cold ethanol fractionation, solvent/detergent inactivation and nanofiltration) are incorporated into the manufacturing process [4-8]. The final product contains $100 \pm 10 \mathrm{mg} / \mathrm{mL}$ protein, sodium chloride, glycine and polysorbate 80 at low $\mathrm{pH}$. The $\mathrm{IgG}$ monomer plus dimer content is $>95 \%$.

Study Patients

Sixty-three patients with primary immunodeficiency were enrolled and treated at 15 study sites. Enrollees were male or female ages $\geq 6$ and $\leq 75$ years with a confirmed diagnosis of primary immunodeficiency, a history of hypogammaglobulinemia (i.e., $\mathrm{IgG}<500 \mathrm{mg} / \mathrm{dL}$ ) or deficient antibody production prior to $\mathrm{IgG}$ replacement therapy. Prior to enrollment, patients were required to have received IVIG infusions every 3 or every 4 weeks for $\geq 3$ months at a dose aimed to be between 300 and $800 \mathrm{mg} / \mathrm{kg}$. Patients were excluded if they were positive at screening for any markers of infectious bloodborne viruses, had a history of adverse reactions to other $\mathrm{IgG}$ or blood products, had selective IgA deficiency or antibodies to $\operatorname{IgA}$, had a history of acute renal failure/or severe renal impairment, had a history of deep venous thrombosis or were pregnant or lactating.

\section{Study Design}

This was an open-label, phase III safety, efficacy and pharmacokinetic study of patients with documented primary immunodeficiency. Each patient was scheduled to receive a Biotest IVIG infusion of 300 to $800 \mathrm{mg} / \mathrm{kg}$ every 3 or 4 weeks for approximately 1 year. Dosing intervals were determined by the patient's pre-study dosing schedule. All patients had safety monitoring studies performed prior to each infusion, at the final clinical visit and 3 months after the last infusion. Blood samples were collected before each infusion for determination of trough $\mathrm{IgG}$ levels. Patients also had blood samples taken prior to several infusions to test for anti-Streptococcus pneumoniae, anti-Haemophilus influenzae type b and anti-tetanus toxoid antibody concentrations.

Twenty patients ( 5 on the 3 week and 15 on the 4 week dosing schedule) were enrolled in the pharmacokinetic (PK) portion of the study. Tests for total $\mathrm{IgG}$, IgG subclasses, Streptococcus pneumoniae antibodies, Haemophilus influenzae type b antibody and tetanus toxoid antibody were performed for PK analysis.

When necessary, doses of Biotest IVIG were adjusted during the study to maintain minimum trough $\mathrm{IgG}$ concentrations $>500 \mathrm{mg} / \mathrm{dL}$.

\section{Evaluation of Safety}

Patients were monitored for adverse events (AEs) and serious adverse events (SAEs) during infusion and between infusions using home diaries. AEs were defined as a treatment-emergent adverse events associated with the use 
of IVIG, whether or not the AE was determined to be product related. The patients recorded all AEs, complaints or problems including start and stop dates, start and stop times and severity in their diaries.

AEs occurring during and within $72 \mathrm{~h}$ of infusion regardless of causality were considered temporally associated adverse events (TAAE). The primary safety endpoint was defined as the proportion of infusions with $\geq 1$ temporally associated AEs including those that were determined to be unrelated to the investigational product. The target for this endpoint was an upper one-sided $95 \%$ confidence limit of less than $0.40[9]$.

\section{Evaluation of Efficacy}

The primary efficacy endpoint was demonstration that the rate of acute serious bacterial infections (SBIs) was less than 1.0 per person-year during regular administration of investigational IVIG for 12 months. Diagnostic criteria for SBIs were defined prospectively [9].

Secondary efficacy parameters included all infections of any kind or severity, time to the first infection of any kind, time to first SBI, days missed from school or work due to infection, days on antibiotics, hospitalizations and days of hospitalization due to infection.

\section{Evaluation of Pharmacokinetics (PK)}

PK assessments were performed at the $4^{\text {th }}$ or $5^{\text {th }}$ infusion of study IVIG in order to wash out previous IVIG products. At certain study sites, PK parameters were assessed at infusion 13 or infusion 17 for patients who did not participate in the earlier PK study. Blood samples for PK assessment were taken before the infusion and at the following times after the infusion: $15 \mathrm{~min}, 1 \mathrm{~h}, 24 \mathrm{~h}, 3$ days, 7 days, 14 days, 21 days and 28 days (if applicable.) The samples were tested for concentrations of total IgG, IgG subclasses and specific antibodies against several $S$. pneumoniae capsular polysaccharide serotypes, $H$. influenzae type $\mathrm{b}$ and tetanus toxoid. The calculated pharmacokinetic parameters were Cmax, the maximum serum concentration, Tmax, the time to reach the maximum serum concentration, AUC 0 -t, the area under the concentration-time curve over 1 dosing interval, $t_{1 / 2}$ or the elimination half-life, CL the total body clearance and Vz, the volume of distribution.

\section{Statistical Analysis}

Descriptive summaries are provided where appropriate for each of the primary and secondary endpoints. In general, summaries are provided for the entire Safety, Intent to Treat (ITT), Per Protocol (PP) or PK populations and by IVIG infusion schedule (i.e., 3-week or 4-week dosing schedule).
Continuous, quantitative, variable summaries include the number of patients $(\mathrm{N})$, mean, standard deviation (SD), median, and range (minimum and maximum). Categorical, qualitative, variable summaries include the frequency and percentage of patients who are in the particular category. In general the denominator for percentage calculations was based upon the number of patients by infusion schedule (i.e., 3-week or 4-week-cycle) or overall, unless otherwise specified.

All efficacy and safety analyses were performed using SAS $^{\circledR}$ Software version 8.2 or later. All pharmacokinetic parameter calculations were performed using WinNonlin Professional ${ }^{\circledR}$ version 5.2 or later but summary statistics were supported by SAS ${ }^{\circledR}$ Software version 8.2 or later.

\section{Results}

Sixty-three patients were enrolled in this study, were treated with Biotest-IVIG and were included in the population evaluated for safety. Seventeen patients were infused with investigational product every 3 weeks and 46 were infused every 4 weeks. Of the 63 enrollees, 5 lost to follow-up when a study site was discontinued. This resulted in 58 patients in the ITT population. Seven patients with major protocol violations were excluded from the ITT population resulting in a per-protocol (PP) population of 51 patients. The ITT and PP populations were analyzed for efficacy.

As shown in Table I, $98 \%$ of patients were Caucasian and the most common immunodeficiency diagnosis was common variable immunodeficiency (CVID) ( $81 \%$ of patients). The average age was 41.2 years (range 6-75). Eight patients had a history of acute serious bacterial infection (SBI) prior to entry into this study. The most common was bacterial pneumonia in 7 patients.

\section{Safety}

During the study a total of 746 infusions of BiotestIVIG were administered to the 63 patients in the safety population with a mean of 13 infusions per patient and a range of 1 to 17 infusions. The mean dose per patient per infusion was $500 \mathrm{mg} / \mathrm{kg}$ with a range of 254 to $1029 \mathrm{mg} / \mathrm{kg}$. Fifty-two patients were treated with study product for 12 months. More than $80 \%$ of patients were infused at a rate $\geq 3.0 \mathrm{~mL} / \mathrm{kg} / \mathrm{h}(300 \mathrm{mg} / \mathrm{kg} / \mathrm{h})$ and $49.6 \%$ were infused at a rate $\geq 3.5 \mathrm{~mL} / \mathrm{kg} / \mathrm{h}(350 \mathrm{mg} / \mathrm{kg} / \mathrm{h})$.

A total of 937 AEs were recorded in the 63 patients in the safety population (Table II). Of the 937 AEs, the most frequent $(>20 \%)$ were headache $(50.8 \%)$, sinusitis $(38.1 \%)$ fatigue $(28.6 \%)$, upper respiratory tract infection $(25.4 \%)$, cough $(22.2 \%)$, pharyngolaryngeal pain and diarrhea (each 
Table I Demographics and baseline characteristics of the safety population

\begin{tabular}{lc}
\hline Parameter & Total $(N=63)$ \\
\hline Gender & \\
Female & $32(50.8 \%)$ \\
Male & $31(49.2 \%)$ \\
Age (yr) & \\
Mean (SD) & $41.2(19.68)$ \\
Median & 44.0 \\
Minimum, Maximum & 6,75 \\
Age group & \\
6-11 Years & $4(6.3 \%)$ \\
12-17 Years & $6(9.5 \%)$ \\
18-64 Years & $44(69.8 \%)$ \\
65 Years and Older & $9(14.3 \%)$ \\
Race & \\
Caucasian & $62(98.4 \%)$ \\
Asian & $1(1.6 \%)$ \\
Primary diagnosis & \\
X-linked agammaglobulinemia & $6(9.5 \%)$ \\
Common variable immunodeficiency & $51(81.0 \%)$ \\
Other hypogammaglobulinemia & $6(9.5 \%)$ \\
SBI history & $8(12.7 \%)$ \\
Bacterial pneumonia & $7(11.1 \%)$ \\
Other & $1(1.6 \%)$ \\
\hline
\end{tabular}

20.6\%), Six patients (9.5\%) reported 339 (36.2\%) of the 937 AEs.

Table II Summary of adverse events in the safety population $(N=63)$

\begin{tabular}{lc}
\hline Metric & Total (\%) \\
\hline Adverse events & 937 \\
Total number of infusions & 746 \\
Patients with $\geq 1 \mathrm{AE}$ & $59(93.7 \%)$ \\
Drug-related AEs & $300(32.0 \%)$ \\
Patients with $\geq 1$ drug-related AE & $40(63.5 \%)$ \\
Infusions with $\geq 1$ TAAE & $209(28.0 \%)$ \\
Infusions with $\geq 1$ drug-related TAAE & $152(20.4 \%)$ \\
Patients with $\geq 1$ SAE & $7(11.1 \%)$ \\
Number of SAEs & 11 \\
Patients with $\geq 1$ drug-related SAE & $1(1.6 \%)$ \\
Patients with $\geq 1$ AE leading to withdrawal & $2(3.2 \%)$ \\
Severity of drug-related AEs & \\
Mild (No. of patients) & $11(17.5 \%)$ \\
Moderate (No. of patients) & $21(33.3 \%)$ \\
Severe (No. of patients) & $8(12.7 \%)$ \\
\hline
\end{tabular}

$T A A E$ temporally associated adverse event, i.e. occurs within $72 \mathrm{~h}$ of an infusion

$S A E$ serious adverse event
Fifty-nine patients (93.7\%) reported at least one adverse event. Of these, 40 patients $(63.5 \%)$ were judged to have experienced an $\mathrm{AE}$ that was related to study product. Mild AEs occurred in $11(17.5 \%)$ patients, moderate AEs occurred in $21(33.3 \%)$ patients and severe AEs were reported in $8(12.7 \%)$ patients. The most frequent severe, drugrelated AEs were headache (3 patients, 4.8\%), migraine and fatigue ( 2 patients, $3.2 \%$ each).

Eleven serious adverse events (SAEs) were reported in 7 (11.1\%) patients. Two of the SAEs (vomiting, mild in severity and dehydration, moderate in severity) leading to hospitalization in 1 patient were considered as related to study product. None of the SAEs resulted in a dose change, dose interruption or discontinuation from the study and all the SAEs resolved. There were no deaths.

There were 431 temporally associated AEs (TAAEs) in $47(74.6 \%)$ patients. Most TAAEs (335, 78\%) occurred during and within the first $24 \mathrm{~h}$ after infusion in 36 (57.1\%) patients. The proportion of infusions with $\geq 1$ temporally associated AEs, regardless of relationship to study product, was $27.7 \%$ with an upper one-sided $95 \%$ confidence limit of $\leq 30.6 \%$. This is significantly below the upper one-sided $95 \%$ confidence limit of $40 \%$ for TAAEs recommended by FDA [9]. The most frequent TAAE reported was headache (occurred with 115 infusions in 27 patients) followed by fatigue (59 infusions in 15 patients) (Table III).

There was no clinically relevant effect of study treatment on systolic blood pressure or diastolic blood pressure measured before or after study infusions. Although several patients had AEs related to vital signs, no safety concerns were identified by the investigators.

There were no hematology or clinical chemistry abnormalities determined by an investigator to be clinically relevant. None of the patients exhibited signs of hemolysis at any time during the study as demonstrated by stable values of hematocrit, hemoglobin and LDH.

Table III Temporally associated adverse events (TAAEs) in more than $5 \%$ patients in the safety population $(N=63)$

\begin{tabular}{lcc}
\hline AE & $\begin{array}{l}\text { By Patient (n, \%) } \\
(47 \text { patients) }\end{array}$ & $\begin{array}{l}\text { By Infusion (n, \%) } \\
\text { (431 infusions) }\end{array}$ \\
\hline Headache & $27(42.9 \%)$ & $115(15.4 \%)$ \\
Fatigue & $15(23.8 \%)$ & $59(7.9 \%)$ \\
Infusion site reaction & $5(7.9 \%)$ & $5(0.7 \%)$ \\
Nausea & $5(7.9 \%)$ & $8(1.1 \%)$ \\
Blood Pressure increased & $4(6.3 \%)$ & $5(0.7 \%)$ \\
Diarrhea & $4(6.3 \%)$ & $4(0.5 \%)$ \\
Dizziness & $4(6.3 \%)$ & $4(0.5 \%)$ \\
Lethargy & $4(6.3 \%)$ & $4(0.5 \%)$ \\
\hline
\end{tabular}


Tests for parvovirus B19, HIV, HCV and HBV were performed at screening, prior to infusions 8 and 12 and at the final safety follow-up visit. There was no evidence of viral transmission by study product. There was a single positive finding for parvovirus B19 during the study that was attributed to exposure to a child with symptomatic Fifth's disease.

\section{Efficacy}

Two serious bacterial infections occurred during the study. A 20 year old man treated every 4 weeks with study IVIG developed pneumonia 16 days after his last study infusion. He was hospitalized and the diagnosis of pneumonia was confirmed by computed axial tomography. The pneumonia resolved 5 days after diagnosis. A 48 year old woman treated every 4 weeks with study IVIG was hospitalized for apparent acute exacerbation of chronic obstructive bronchitis that was later classified by the FDA as bacterial pneumonia. These two episodes resulted in a serious bacterial infection rate of 0.035 SBI/patient/year with an upper $99 \%$ confidence limit of $\leq 0.136 \mathrm{SBI} /$ patient/year. This result is substantially below the target SBI rate of $\leq 1.0 \mathrm{SBI} /$ patient/year defined by the FDA as an appropriate endpoint for PID patients receiving regular doses of IVIG [9].

Infections of any kind or seriousness are listed in Table IV. Since the incidence of infections was essentially the same in the PP population as in the ITT population, the most frequent infections (other than SBI) were reported for the ITT population (Table V) and included acute sinusitis, other respiratory tract infections, unclassified infections designated as "other," otitis media, and bronchitis.

Table VI lists the secondary efficacy endpoints observed in the ITT population. Means, standard deviations and rates are summarized. Twenty-one patients (33.3\%) missed work or school because of infection at a rate of 2.28 days/patient/ year (Table VI). Thirty-eight patients $(60.3 \%)$ were treated with therapeutic antibiotics at a rate of 39.1 days of therapeutic antibiotics/patient/year. The rate of hospitalizations was 0.21 days per patient per year.

Table IV Infections of any kind or seriousness in the ITT and PP populations

\begin{tabular}{lll}
\hline Parameter & ITT $(N=58)$ & PP $(N=51)$ \\
\hline Total Infections & 139 & 132 \\
Mean \pm SD per subject & $2.4 \pm 2.7$ & $2.6 \pm 2.7$ \\
$\begin{array}{l}\text { Infections per subject per } \\
\text { year (90\% CI) }\end{array}$ & $2.6[2.3-2.7]$ & $2.6[2.2-3.0]$ \\
\hline
\end{tabular}

Table $\mathbf{V}$ Infections observed in the ITT population $(N=58)$

\begin{tabular}{lc}
\hline Infections & $\begin{array}{l}\text { No. of Patients } \\
\text { (\% of infections) }\end{array}$ \\
\hline Acute sinusitis & $36(25.9 \%)$ \\
Other respiratory tract infection & $30(21.6 \%)$ \\
Other & $23(16.5 \%)$ \\
Otitis Media/ear infection & $15(10.8 \%)$ \\
Bronchitis & $14(10.1 \%)$ \\
Acute exacerbation of chronic sinusitis & $7(5.0 \%)$ \\
GI Infection & $7(5.0 \%)$ \\
Urinary tract infection & $4(2.9 \%)$ \\
Pneumonia & $2(1.4 \%)$ \\
Conjunctivitis & $1(0.7 \%)$ \\
\hline
\end{tabular}

Pharmacokinetics

The PK population was composed of 5 patients in the 3 -week infusion group and 16 in the 4-week group. PK parameters for total IgG are shown in Table VII. Cmax and Tmax were similar for the 2 treatment groups. The area under the concentration-time curve AUC 0-t was higher in 4-week patients than 3-week patients because of the longer time

Table VI Secondary efficacy endpoints in the ITT population

\begin{tabular}{ll}
\hline Parameter & Total $(N=58)$ \\
\hline Infections of any kind (total) & 139 \\
Mean \pm SD & $2.4 \pm 2.7$ \\
Min-Max & $0-14$ \\
Patients with any infection (n) & 40 \\
Infection rate/patient/year & 2.6 \\
Days off work/school due to infection (n) & \\
Mean \pm SD & $2.1 \pm 4.84$ \\
Min - Max & $0-24$ \\
Patients with any days off work or school (n) & 21 \\
Days off work or school/patient/year & 2.28 \\
Days on antibiotics & \\
Mean \pm SD & $76.4 \pm 118.3$ \\
Min - Max & $0-372$ \\
Patients with any days on antibiotics (n) & 46 \\
Days of antibiotics/patient/year & 82 \\
Days on therapeutic antibiotics & \\
Mean \pm SD & $36.2 \pm 52.7$ \\
Min - Max & $0-306$ \\
Patients on therapeutic antibiotics (n) & 38 \\
Days of therapeutic antibiotics/patient/year & 39.1 \\
Days of hospitalization due to infection (n) & 11 \\
Patients hospitalized (n) & 2 \\
Hospitalization days/patient/year & 0.21 \\
\hline
\end{tabular}

$S D$ standard deviation 
Table VII IgG pharmacokinetic parameters

\begin{tabular}{lll}
\hline Parameter & $\begin{array}{l}\text { 3-week cycle }(N=5) \\
\text { Mean } \pm \mathrm{SD})\end{array}$ & $\begin{array}{l}\text { 4-week cycle }(N=16) \\
\text { Mean } \pm \mathrm{SD})\end{array}$ \\
\hline $\mathrm{C}_{\max }(\mathrm{mg} / \mathrm{dL})$ & $2184 \pm 293$ & $2122 \pm 425$ \\
$\mathrm{~T}_{\max }$ median & $4.05(2.67-26.1)$ & $3.48(2.6-78.6)$ \\
$\quad($ range) (h) & $668,173 \pm 118,198$ & $852,213 \pm 155,334$ \\
$\begin{array}{c}\mathrm{AUC} 0-\mathrm{t} \\
\left(\mathrm{h}^{*} \mathrm{mg} / \mathrm{dL}\right)\end{array}$ & $19.6(4.1)$ & $33.5(10.7)$ \\
$\mathrm{T}_{1 / 2}(\mathrm{days})$ & $(16.2-26.7)$ & $(18.3-51.6)$ \\
$\quad(\mathrm{min}-\mathrm{max})$ & $1.97 \pm 0.22$ & $1.41 \pm 0.46$ \\
$\mathrm{CL}(\mathrm{mL} / \mathrm{kg} / \mathrm{day})$ & $0.056 \pm 0.014$ & $0.064 \pm 0.015$ \\
$\mathrm{Vz}(\mathrm{L} / \mathrm{kg})$ & & \\
\hline
\end{tabular}

interval between infusions. The IgG half-life in 3-week subjects was 19.6 days compared to 33.5 days in 4-week subjects; however these differences were not significant.

Pharmacokinetic parameters of specific antibodies were comparable to values for total IgG. Data from 28-day patients are shown in Table VIII. Similar results were observed in the five 21 day patients (data not shown). The antibody half-lives are the same order of magnitude as total $\mathrm{IgG}$ with values ranging from 25 to 84 days.

The doses administered in this study produced mean trough IgG levels of $1076 \pm 254 \mathrm{mg} / \mathrm{dL}$ (range 606 to $1780 \mathrm{mg} / \mathrm{dL}$ ) in 21-day patients and $943 \pm 215$ (range 487 to $2250 \mathrm{mg} / \mathrm{dL}$ ) in 28 -day patients. The $487 \mathrm{mg} / \mathrm{dL}$ value was a single test result that was reported at the $6^{\text {th }}$ infusion in one patient; however, trough IgG levels above $500 \mathrm{mg} / \mathrm{dL}$ were observed at all other infusions in this patient.

\section{Discussion}

Biotest-IVIG incorporates the best practices in IVIG manufacturing by combining Cohn-Oncley cold ethanol fractionation, purification by ion-exchange chromatography, solventdetergent virus inactivation, virus removal by nanofiltration and formulation at $10 \%$ protein $[2-8]$.
The purpose of this clinical trial was to evaluate the safety, efficacy, tolerability and pharmacokinetics of Biotest-IVIG in patients with primary immunodeficiency. The study trial followed the FDA guidelines published in 2008 that has lead to the standardization of many safety, efficacy and pharmacokinetic endpoints [9].

The observed types, severity and frequency of AEs assessed as medically related to Biotest-IVIG are commonly reported as associated with infusions of any IVIG. Eighty percent of the adverse events related to study IVIG were judged to be mild or moderate. As reported in other IVIG clinical trials [10-15], the most common adverse event was headache. A large proportion of adverse events $(339,36.2 \%)$ occurred in a small number of patients (6 patients, 9.5\%). Although sporadic fluctuations in blood pressure and pulse were noted throughout the study, none were considered to be clinically significant. Analyses of serum chemistries, urinalysis, and viral safety tests showed no clinically significant or unexpected results for a population of PID patients receiving IVIG.

The primary efficacy endpoint, $0.035 \mathrm{SBI} /$ person/year, compares favorably to SBI rates observed in studies of other IVIGs in patients with primary immunodeficiency who received IVIG doses that were similar to those administered in this study [10-15].

Infections other than serious infections occurred at a rate of $2.6 \mathrm{infections/patient/year.} \mathrm{The} \mathrm{most} \mathrm{frequent} \mathrm{infections}$ were acute sinusitis and other respiratory tract infections.

Among other efficacy parameters, the rate of days away from work or school because of infection (2.28 days/patient/ year) was relatively low compared to literature reports. [10-14]. Analysis of days on antibiotics revealed that investigators were more likely to treat patients on 21-day infusion schedules with prophylactic antibiotics than patients treated with IVIG every 28 days. This correlation between three week IVIG dosing and prophylactic antibiotic treatment may reflect difficulty in controlling infection in this patient population.

All patients maintained average trough IgG concentrations above the target level of $>500 \mathrm{mg} / \mathrm{kg}$ with higher mean trough levels observed in recipients of infusions every 21 days

Table VIII Pharmacokinetic parameters of specific antibodies in patients infused every 4 weeks

\begin{tabular}{|c|c|c|c|c|c|}
\hline & \multirow{2}{*}{$\begin{array}{l}\text { Tetanus }(N=16) \\
(\mathrm{IU} / \mathrm{mL})\end{array}$} & \multirow{2}{*}{$\begin{array}{l}\text { H. influenzae b }(N=15) \\
(\mathrm{mg} / \mathrm{L})\end{array}$} & \multicolumn{3}{|c|}{ S. pneumoniae serotypes $(\mu \mathrm{g} / \mathrm{mL})(N=16)$} \\
\hline & & & 14 & $19 \mathrm{~A}$ & $23 \mathrm{~F}$ \\
\hline Elimination half-life (days) & $83.76(n=14)$ & $25.16^{\mathrm{a}}(n=14)$ & $40.77(n=15)$ & $60.04(n=15)$ & $29.98(n=14)$ \\
\hline Cmax (units) & 6.96 & 6.62 & 14.62 & 46.14 & 36.38 \\
\hline Tmax (days) & 0.131 & 0.644 & 1.821 & 1.861 & 1.682 \\
\hline $\mathrm{AUC}_{0-\mathrm{t}}$ (units*days) & $142.48(n=14)$ & $96.09(n=14)$ & $253.99(n=15)$ & $1026.63(n=15)$ & $563.89(n=15)$ \\
\hline
\end{tabular}

${ }^{a}$ Outlying value of patient 6003 was excluded from calculation. 
compared to patients who received infusions every 28 days. In general, concentration-time profiles were similar for $\operatorname{IgG}, \operatorname{IgG}$ subclasses and specific antibodies with initial increases on the day of the infusion followed by slow return to baseline at around 14 to 21 days (data not shown).

Half-life results for total $\mathrm{IgG}$ and specific antibodies demonstrated significant interpatient variability. The IgG half-life for all patients was approximately 30 days with a $37.5 \%$ coefficient of variation. Variation in half-life determinations may reflect shortcomings associated with measuring the decline in concentration of $\operatorname{IgG}$ [16]. If the rate of elimination depends on serum concentration, it is possible that half-lives are altered by different doses.

The differences in pharmacokinetic parameters resulted from differences in individual dosing since doses were titrated for each subject. AUC0-t is always larger for the 4-week period compared to the 3 -week period unless the dose is reduced, otherwise the patient is receiving more IgG.

Differences in AUC0-t result in differences calculated for $\mathrm{CL}$ and Vz. Total body clearance is dose divided by AUC0-t so the difference in $\mathrm{AUC} 0$-t produced a difference in total body clearance. Volume of distribution is CL divided by the elimination rate constant. Individual doses were used for calculation of CL. Therefore dose differences also contribute to $C L$ and $\mathrm{Vz}$ variation.

Half-life variability may also result from differences in each patient's ability to synthesize endogenous IgG. If a patient produces IgG, the serum concentration is increased and the half-life appears to be prolonged [16].

Measurement of passively acquired antibodies to specific organisms may provide more meaningful clinical information about $\mathrm{IgG}$ metabolism than determination of $\mathrm{IgG}$ concentrations by immunochemical tests if the antibodies are not consumed by an infection. Unfortunately antibody levels are extremely low (micrograms or nanograms per $\mathrm{mL}$ ) compared to $\operatorname{IgG}(\mathrm{mg} / \mathrm{mL})$ and it is often difficult to accurately measure levels above baseline especially $2-4$ weeks after infusion. In this study, determination of the pharmacokinetic parameters for several representative antibodies indicated that antibody activities that are present in the starting plasma are preserved during the manufacture of Biotest-IVIG.

Acknowledgements Financial support was provided by Biotest Pharmaceuticals Corporation, Boca Raton, Florida. The authors wish to thank the following persons from Biotest Pharmaceuticals Corporation for their support and successful completion of the study through study management and review of the manuscript: Shailesh Chavan and Debbie L. Mirjah. We also wish to thank the following persons from Biotest AG, Dreieich, Germany for their support in review of the manuscript: Andrea Wartenberg-Demand and Rainer Schmeidl. The statistical support provided by Premier Research Group and Mathias Broz (StatConsult, Magdeburg, Germany) and the technical support provided by John Hooper (BioCatalyst Research LLC, Liberty, Missouri, USA) is gratefully appreciated.
Open Access This article is distributed under the terms of the Creative Commons Attribution License which permits any use, distribution, and reproduction in any medium, provided the original author(s) and the source are credited.

\section{References}

1. Bruton OC. Agammaglobulinema. Pediatrics. 1952;9:722-7.

2. Cohn EJ, Strong LE, Hughes Jr WL, et al. Preparation and properties of serum and plasma proteins III: A system for the separation into fractions of the protein and lipoprotein components of biological tissues and fluids. J Am Chem Soc. 1946;68:459-75.

3. Melin JL, Oncley M, Richert DA, et al. The separation of the antibodies, isoagglutinins, prothrombin, plasminogen and betalipoprotein into subfractions of human plasma. J Am Chem Soc. 1949;71:541-50.

4. Wells MA, Wittek AE, Epstein JS, Marcus-Sekura C, Daniel S, Tankersley DL, et al. Inactivation and partition of human T-cell lymphotrophic virus, type III, during ethanol fractionation of plasma. Transfusion. 1986;6:210-3.

5. Mitra G, Wong MF, Mozen MM, McDougal JS, Levy JA. Elimination of infectious retroviruses during preparation of immunoglobulins. Transfusion. 1986;26:394-7.

6. Yei S, Yu MW, Tankersley DL. Partitioning of hepatitis C virus during Cohn-Oncley fractionation of plasma. Transfusion. 1992;32:824-8

7. Horowitz B, Wiebe ME, Lippin A, Stryker MH. Inactivation of viruses in labile blood derivatives I: Disruption of lipid-enveloped viruses by tri (n-butyl) phosphate detergent combinations. Transfusion. 1985;25:516-22.

8. Omar A, Kempf C. Removal of neutralized model parvoviruses and enteroviruses in human IgG solutions by nanofiltration. Transfusion. 2002;42:1005-10.

9. FDA/CBER. Guidance for industry: Safety, efficacy, and pharmacokinetic studies to support marketing of immune globulin intravenous (Human) as replacement therapy for primary humoral immunodeficiency, http://www.fda.gov.cber/gdlns/igivimmuno. htm, June 2008.

10. Ochs HD, Pinciaro PJ, et al. Octagam ${ }^{\circledR} 5 \%$, an Intravenous IgG Product, is efficacious and well tolerated in patients with primary immunodeficiency diseases. J Clin Immunol. 2004;24(3):309-14.

11. Berger M, Pinciaro PJ, et al. Safety, efficacy and pharmacokinetics of flebogamma ${ }^{\circledR} 5 \%$ [Immune Globulin Intravenous (Human)] for replacement therapy in primary immunodeficiency diseases. J Clin Immunol. 2004;24(4):389-96.

12. Berger M, et al. A multicenter, prospective, open label, historically controlled clinical trial to evaluate efficacy and safety in primary immunodeficiency diseases (PID) patients of Flebogamma ${ }^{\circledR} 5 \%$ DIF, the next generation of Flebogamma. J Clin Immunol. 2007;27:628-33.

13. Church JA, Leibl H, Stein MR, Melamed IR, Rubinstein A, Schneider LC, et al. Efficacy, safety and tolerability of a new $10 \%$ liquid intravenous immune globulin (IGIV 10\%) in patients with primary immunodeficiency. J Clin Immunol. 2006;26:388-95.

14. Stein MR, Nelson RP, Church JA, Wasserman RL, Church JA, Wasserman RL, et al. Safety and efficacy of Privigen ${ }^{\circledR}$, a novel $10 \%$ liquid immunoglobulin preparation for intravenous use, in patients with primary immunodeficiencies. J Clin Immunol. 2009;29:137-44.

15. Berger M, Pinciaro PJ, Althaus A, Ballow M, Chouksey A, Moy J, et al. Efficacy, pharmacokinetics, safety, and tolerability of flebogamma ${ }^{\circledR} 10 \%$ DIF, a high-purity human intravenous immunoglobulin, in primary immunodeficiency. J Clin Immunol. 2010;30:321-9.

16. Schiff RI. Intravenous gammaglobulin: pharmacology, clinical uses and mechanisms of action. Pediatr Allergy Immunol. 1994;5:63-87. 